\title{
Clinical and molecular correlates from a predominantly adult cohort of patients with short telomere lengths
}

(c) The Author(s) 2021

\author{
Blood Cancer Journal (2021)11:170; https://doi.org/ \\ 10.1038/s41408-021-00564-7
}

\section{Dear Editor}

Telomere biology disorders (TBDs) are accelerated aging syndromes affecting hematopoietic, pulmonary, hepatobiliary, and/or immunological systems among others [1, 2]. Adult-onset TBDs are commonly associated with pathogenic variants in TERT and TERC, and manifest with varying degree of organ involvement such as idiopathic interstitial pneumonia (IIP), cryptogenic cirrhosis, unexplained bone marrow failure (BMF) and/or immunodeficiency, and an inherent risk for cancer development such as myeloid leukemias and squamous cell carcinomas among others [3-9]. Though fluorescence in-situ hybridization (FlowFISH)determined age-adjusted lymphocyte $T L>50 \%$ tile have a $100 \%$ negative predicted value for variant detection, the TL threshold below which genetic screening should be pursued (especially in older patients) is still not known $[10,11]$. The optimal TL threshold for variant detection depends on age, with individuals at ages $<20$ years showing strong correlations with lower $\mathrm{TL}$, while the $\mathrm{TL}$ in older variant carriers often overlaps with the lower decile of normal controls [10]. Genetic screening is suggested for $T L \leq 10$ th centile in lymphocytes or granulocytes, especially in the presence of suggestive personal or family history of a TBD [1]. However, the utility of genetic testing for adult patients with TL $>10$ th centile and integration of clinical phenotype is currently unclear. Additionally, natural outcomes and diagnostic algorithms are less clear in adult (age $\geq 18$ years) patients with short telomeres (defined as $T L \leq 10$ th centile in lymphocytes).

We retrospectively abstracted data from patients who underwent FlowFISH testing at our institution from years 2015 to 2020. All patients who underwent FlowFISH testing had a suspected clinical phenotype based on at least one or more of the salient clinical features mentioned below. FlowFISH assessments were done at reference laboratories in Vancouver (RepeatDx; Canada) and Johns Hopkins University (JHU, USA). In order to objectively quantify clinical risk, a clinical likelihood score (CLS) was developed based on the number of presenting clinical features suggestive of TBD (prior to FlowFISH testing), or the presence of a significant family history of the same in one or more 1 st or 2 nd degree relatives, with categories including low (none or 1), intermediate (2), or high risk $(>2)$, respectively (Supplementary Methods). Salient clinical features were pre-determined as, personal history of premature hair graying (onset at age $<30$ years), idiopathic interstitial pneumonia (IIP), or IIP/emphysema overlap, cryptogenic cirrhosis or nodular regenerative hyperplasia $(\mathrm{NRH})$, persistent unexplained cytopenias [defined as low blood counts in one or more cell lineages (red or white blood cell or platelets), persistent for 6 months or longer], and/or immunodeficiency.
Genetic testing was performed using either an in-house research-based whole exome sequencing (WES) or commercial bone marrow failure-specific targeted next generation sequencing (NGS) panel or exome-based customized panels (ES-Slices) (Supplementary table 1). Whole-exome sequencing (WES) was performed at the Clinical Genomics Laboratory (Mayo Clinic) using a previously published protocol $[12,13]$. Genomic data was processed through an in-house bioinformatics pipeline and analyzed by the Translational Omics Program at the Center for Individualized Medicine (Mayo Clinic) using Emedgene analysis software (Emedgene Technologies).

Two hundred and fifty-two patients at our institution underwent TL assessment at RepeatDx $(n=71)$ and JHU $(n=181)$ laboratories. Median age was 57 (range: 4-83) years; 144 (57\%) being males; 236 (94\%) adults. Significant family history was present in $66(26 \%)$ patients, while premature graying of hair was present in $24(10 \%)$ patients. Organ-specific clinical features included cytopenias $(n=117,46 \%)$, IIP $(n=135,54 \%)$, gastrointestinal disease $[n=38(15 \%)$, cryptogenic cirrhosis-30, NRH-4, enteropathy-2, steatosis-2], and immunodeficiency $(n=37,15 \%)$. Patterns of IIP included 41 (30\%) usual interstitial pneumonia (UIP), 15 (11\%) non-specific interstitial pneumonia (NSIP), 9 (7\%) chronic hypersensitivity pneumonitis (CHP), 2 (1.5\%) lymphocytic interstitial pneumonitis and 1 desquamative interstitial pneumonia. History of smoking was present in 80 (32\%) patients, with a median of $24(0.5-210)$ pack years of smoking and correlated with IIP $(p=0.01)$. Emphysematous changes were seen in $15(11 \%)$ patients; $67 \%$ in smokers [median, 29 (2.5-70) pack years]. Pulmonary function test information was available in 148 [59\%, $108(80 \%)$ in patients with pulmonary disease]. The \%predicted values of FEV1 [median, 63.5 versus 92, $P<0.0001$ ] and FVC [median 61.1 versus $98.5, P<0.0001$ ] were lower, while the FEV1/ FVC ratio (median 106.7 versus 93.7, $P<0.0001$ ) was higher in patients with versus without IIP [defined by high-resolution computed tomography (HRCT) imaging findings [14]]. The clinical diagnoses for patients before TL testing included 107 (42\%) IIP, 22 (9\%) aplastic anemia, $13(5 \%)$ each with unexplained pancytopenia, immunodeficiency, and IIP/cirrhosis, 12 (5\%) cirrhosis, 11 (4\%) common variable immunodeficiency (CVID), 8 (3\%) each with BMF and lymphopenia, 5 (2\%) each with unexplained anemia, bicytopenia, and myelodysplastic syndrome, 4 (2\%) each with IIP/CVID and neutropenia, 3 (1\%) with thrombocytopenia, $2(<1 \%)$ each with hypersensitivity pneumonitis (HP) and NRH, $1(<1 \%)$ each with CVID/NRH, acute myeloid leukemia, Diamond-Blackfan Anemia, IIP/BMF, IIP/cirrhosis/BMF, IIP/NRH/BMF, IIP/pancytopenia, and leukopenia. Seven (3\%) patients were tested only due to a significant family history without an obvious clinical phenotype.

CLS stratification included low $(n=139,55 \%)$, intermediate ( $n=83,33 \%)$, and high $(n=30,12 \%)$ groups, with higher CLS significantly correlating with lower delta (age-adjusted) TL for lymphocytes $(P=0.01)$ but not granulocytes $(P=0.1)$. Genetic testing was performed in $82(33 \%)$ patients (targeted NGS panel- 


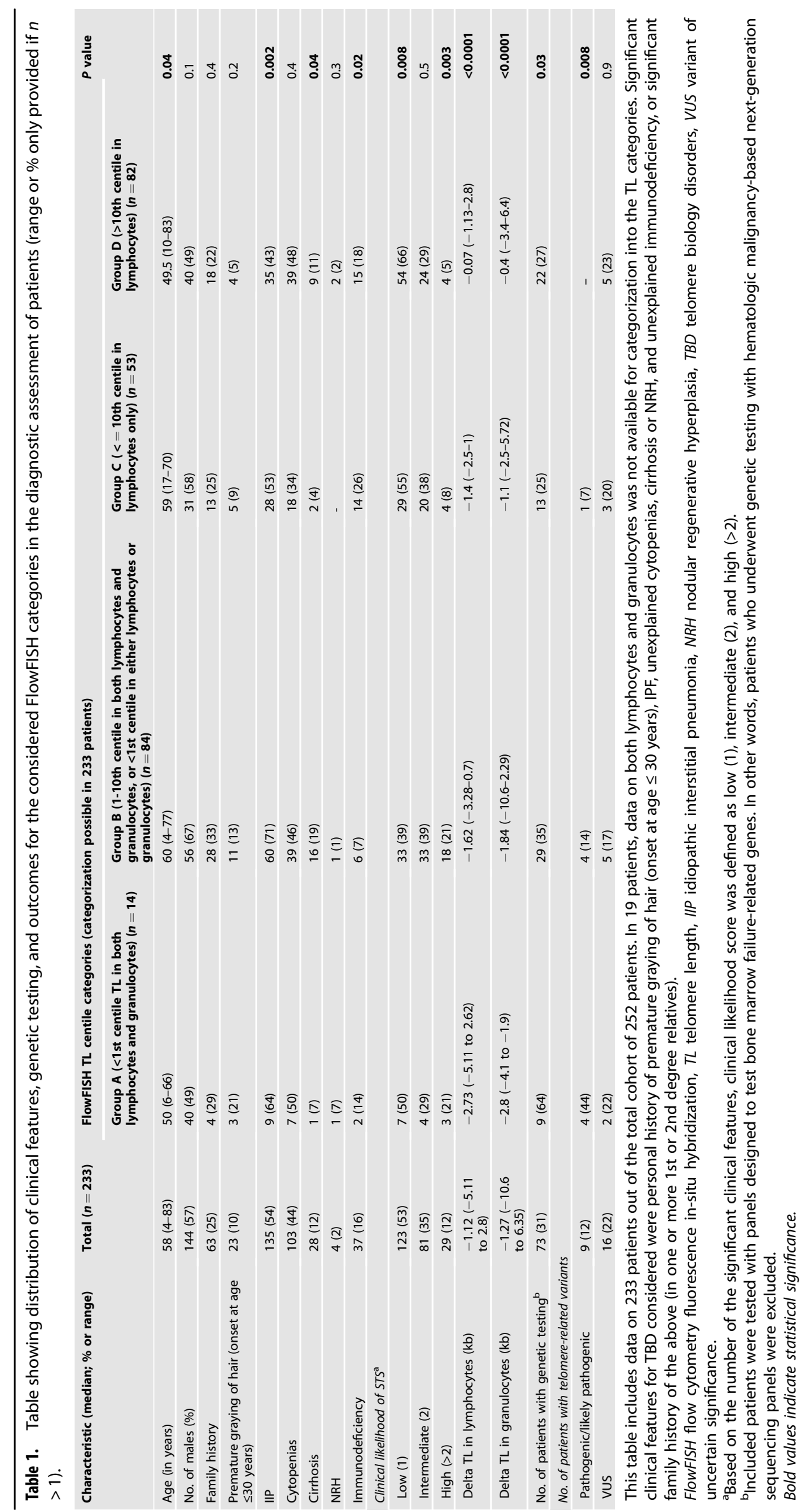


A

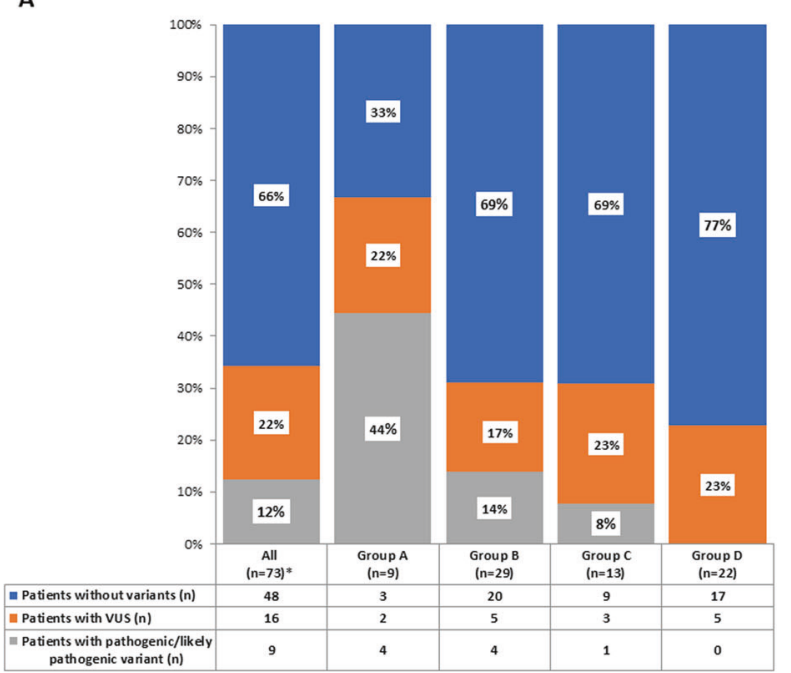

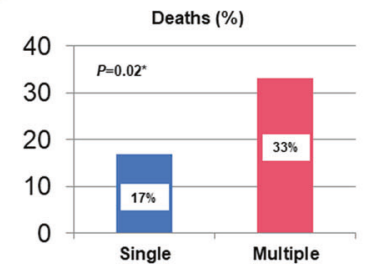

C

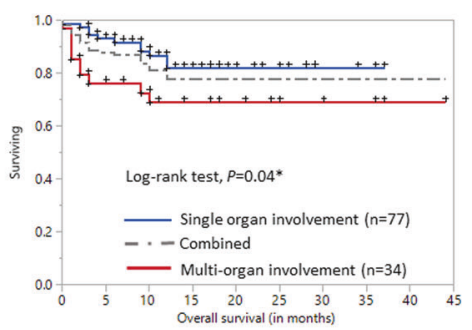

Fig. 1 Figure showing genetic characteristics and clinical outcomes of patients with clinically-relevant short telomeres. A shows genetic testing information and the frequencies of pathogenic variants and variants of uncertain significance in different FlowFISH centile categories. B shows a higher frequency of deaths in patients with multiple organ involvement compared to single organ involvement (33\% versus $17 \%$, $P=0.02^{*}$ ) in patients with clinically-relevant short telomeres (telomere length $\leq 10^{\text {th }}$ centile in lymphocytes). C shows a higher Kaplan-Meier estimate of overall survival (OS) in untreated (non-transplanted) patients with short telomeres (telomere length $\leq 10$ th centile in lymphocytes) with single organ $(n=77)$ versus patients with multiple organ $(n=34)$ involvement (median not reached in either category, $\left.{ }^{*} P=0.04\right)$.

31, ES-Slice-39,WES-12) which was positive for 9 (11\%) pathogenic or likely pathogenic (8 TERT and 1 RTEL1) variants and $16(19.5 \%)$ variants of uncertain significance (Supplementary table 2). Since this was a retrospective study, the genetic analyses are limited by the fact that certain patients were more likely to be tested than others based on clinical features. Among patients with short telomeres (TL in lymphocytes $\leq 10 \%, n=158,146$ adults), only $17 \%$ (9/54 tested patients) were positive for pathogenic variants. This suggests potential alternative, epigenetic or non-genetic mechanisms of telomere shortening/telomere length regulation such as aging-associated methylation and/or chromatin modifications, and environmental factors as suggested in other studies $[15,16]$. 'High risk' per CLS score (as defined above) was unable to predict likelihood of finding a telomere-associated variant $(P=0.6)$, highlighting the shortcomings in the predictive value of phenotypic changes/family histories in adult patients with suspected TBDs.

Based on age-appropriate centile categorization of lymphocyte and granulocyte TL (information for both available in 233 patients), patients were stratified into four groups (Table 1). Group A $(n=14)$ included TL $<1$ st centile in both lymphocytes and granulocytes. Among the 9 (64\%) patients who underwent genetic testing (4 targeted NGS panels, 4 ES-Slice, and 1 WES) in this group, $4(44 \%)$ patients were found to have pathogenic variant (all TERT) and $2(22 \%)$ patients were detected to have a VUS (both RTEL1). Group B included patients with TL 1-10th centile in both lymphocytes and granulocytes, or $<1$ st centile in either lymphocytes or granulocytes $(n=84)$. Twenty-nine $(35 \%)$ underwent genetic testing (11 targeted NGS panels, 16 ES-Slice, and 2 WES) with 4 pathogenic variants ( 3 TERT, 1 RTEL 1), and 5 VUS (2 RTEL 1, 1 TERT, 1 NHP2, and 1 PARN). Group C included patients with TL 1-10th centile in lymphocytes only and $>10$ th centile in granulocytes $(n=53)$. Thirteen $(25 \%)$ patients in this group underwent genetic testing (2 NGS-panel, 7 ES-Slice, 4 WES) with 1 pathogenic variant (TERT) and 3 VUS (2 in TINF2, 1 in RTEL1). Group D included patients with TL $>10$ th centile in lymphocytes $(n=82)$. Genetic testing was done in $22(27 \%)$ patients (9 NGSPanel, 8 ES-Slices, and 5 WES) of whom only 5 (23\%) had VUS in 2 RTEL1,1 TINF2, 1 WRAP53, and 1 CTC1, without any bonafide pathogenic variants (Fig. $1 \mathrm{~A}$ and Table 1). In patients with pulmonary disease, \%predicted values of FEV1 $\left(R^{2}=0.08, P=0.3\right)$,
FVC $\left(R^{2}=0.1, P=0.2\right)$, and FEV1/FVC $\left(R^{2}=0.1, P=0.3\right)$ did not correlate with the likelihood of finding a telomere-associated pathogenic variant. Interestingly, one 47-year-old patient had FlowFISH testing (at the same laboratory) at three different time points in a span of 6 months (post-transplant), with results demonstrating variability in telomere length measurement (Supplementary table 3) and categorization, which could partly be due to the interassay coefficient of variation for FlowFISH $(2.5 \%$ for lymphocytes and $2.1 \%$ for granulocytes) [10]. For practical clinical decision-making, data for additional categories such as patients with $\mathrm{TL}<1$ st centile in lymphocytes and $>1$ st centile in granulocytes, and $1-10$ th centile in lymphocytes, $\geq 1$ st centile in granulocytes is provided in Supplementary table 4 .

We then assessed outcomes for patients with $\mathrm{TL} \leq 10$ th centile in lymphocytes $(n=151)$. At a median follow-up of $15(95 \% \mathrm{Cl}$ 13-17) months, 124 (78\%) patients were alive with 34 (22\%) deaths; higher frequency in non-transplanted (untreated) patients with multiple organ involvement compared with single organ involvement ( 33 versus $17 \%, P=0.02$, Fig. 1B). The median Kaplan-Meier estimate of overall survival computed from the time of FlowFISH testing was not reached. When patients were categorized by organ involvement, those with multiple organ involvement had a worse Kaplan-Meier estimate of overall survival (OS, median not reached, $P=0.04)$, with similar findings for untreated $(n=115)$ patients (median not reached, $P=0.03$, Fig. 1C) and no differences among patients who underwent organ-specific transplantation (supplementary fig. 1).

Our study demonstrates the importance of using a Flow-FISH assay based predictive algorithm to screen adult patients with suspected STS for telomere-related genetic alternations. We also demonstrate the limited role for genetic testing in adult patients with lymphocyte $T L>10$ th centile, regardless of the clinical likelihood. Adult patients with shortened telomere lengths have a $<20 \%$ positivity rate for a TBD-associated pathogenic variant suggesting alternative, epigenetic and/or non-genetic mechanisms of telomere length regulation. Potential caveats of using FlowFISH testing include interassay variability, measuring only mean $\mathrm{TL}$, lack of ability in measuring tissue-specific $T L$, and possibility of missing silent genetic carriers who may have TL at the lower end of the normal range. Patients with multiple organ involvement clearly have worse 
outcomes, with multiorgan transplant strategies available at select centers.

Abhishek A. Mangaonkar (iD ${ }^{1,9}$, Alejandro Ferrer (iD ${ }^{2,9}$, Filippo Pinto E. Vairo ${ }^{2,3}$, Caleb W. Hammel ${ }^{2}$, Carri Prochnow ${ }^{\prime}$, Naseema Gangat $\mathbb{D}^{1}{ }^{1}$, William J. Hogan (D) ${ }^{1}$, Mark R. Litzow (D) ${ }^{1}$, Steve G. Peters ${ }^{4}$, J. P. Scott ${ }^{4}$, James P. Utz ${ }^{4}$, Misbah Baqir ${ }^{4}$, Eva M. Carmona-Porquera ${ }^{4}$, Sanjay Kalra ${ }^{4}$, Hiroshi Sekiguchi ${ }^{4}$, Shakila P. Khan ${ }^{5}$, Douglas A. Simonetto ${ }^{6}$, Eric W. Klee' ${ }^{2}$, Patrick S. Kamath ${ }^{6}$, Anja C. Roden ${ }^{7}$, Avni Y. Joshi ${ }^{8}$, Cassie C. Kennedy ${ }^{4}$, Mark E. Wylam ${ }^{4}$ and Mrinal M. Patnaik (iD) ${ }^{1 凶}$

${ }^{1}$ Division of Hematology, Department of Medicine, Rochester, MN, USA. ${ }^{2}$ Center for Individualized Medicine, Department of Quantitative Health Science, Rochester, MN, USA. ${ }^{3}$ Department of Clinical Genomics, Rochester, MN, USA. ${ }^{4}$ Division of Pulmonary and Critical Care Medicine, Department of Medicine, Rochester, MN, USA.

${ }^{5}$ Division of Pediatric Hematology/Oncology, Department of Pediatrics, Rochester, MN, USA. ${ }^{6}$ Division of Gastroenterology, Department of Medicine, Rochester, MN, USA. ${ }^{7}$ Department of Laboratory Medicine and Pathology, Rochester, MN, USA. ${ }^{8}$ Division of Pediatric Allergy and Immunology, Department of Pediatrics, Mayo Clinic, Rochester, MN, USA. ${ }^{9}$ These authors contributed equally: Abhishek A. Mangaonkar, Alejandro Ferrer ${ }^{\bowtie}$ email: patnaik. mrinal@mayo.edu

\section{REFERENCES}

1. Townsley DM, Dumitriu B, Young NS. Bone marrow failure and the telomeropathies. Blood. 2014;124:2775-83.

2. Heiss NS, Knight SW, Vulliamy TJ, Klauck SM, Wiemann S, Mason PJ, et al. X-linked dyskeratosis congenita is caused by mutations in a highly conserved gene with putative nucleolar functions. Nat Genet. 1998;19:32-38.

3. Calado RT, Young NS. Telomere diseases. New Engl J Med. 2009;361:2353-65.

4. Mangaonkar AA, Ferrer A, Pinto E Vairo F, Cousin MA, Kuisle RJ, Klee EW, et al. Clinical correlates and treatment outcomes for patients with short telomere syndromes. Mayo Clinc Proc. 2018;93:834-9.

5. Mangaonkar AA, Patnaik MM. Short telomere syndromes in clinical practice: bridging bench and bedside. Mayo Clin Proc. 2018;93:904-16.

6. Feurstein S, Adegunsoye A, Mojsilovic D, Vij R, West DePersia AH, Rajagopal PS, et al. Telomere biology disorder prevalence and phenotypes in adults with familial hematologic and/or pulmonary presentations. Blood Adv. 2020;4:4873-86.

7. Armanios MY, Chen JJ, Cogan JD, Alder JK, Ingersoll RG, Markin C, et al. Telomerase mutations in families with idiopathic pulmonary fibrosis. New Engl J Med. 2007;356:1317-26.

8. Calado RT, Regal JA, Kleiner DE, Schrump DS, Peterson NR, Pons V, et al. A spectrum of severe familial liver disorders associate with telomerase mutations. PLoS ONE. 2009;4:e7926.

9. Alter BP, Giri N, Savage SA, Rosenberg PS. Cancer in the National Cancer Institute inherited bone marrow failure syndrome cohort after fifteen years of follow-up. Haematologica. 2018;103:30-39.

10. Alder JK, Hanumanthu VS, Strong MA, DeZern AE, Stanley SE, Takemoto CM, et al. Diagnostic utility of telomere length testing in a hospital-based setting. Proc Natl Acad Sci USA. 2018;115:E2358-e2365.

11. Baerlocher GM, Vulto I, de Jong G, Lansdorp PM. Flow cytometry and FISH to measure the average length of telomeres (flow FISH). Nat Protoc. 2006;1:2365-76.

12. Ferrer A, Mangaonkar AA, Stroik S, Zimmermann MT, Sigafoos AN, Kamath PS, et al. Functional validation of TERT and TERC variants of uncertain significance in patients with short telomere syndromes. Blood Cancer J. 2020;10:120.
13. Mangaonkar AA, Ferrer A, Pinto E Vairo F, Cousin MA, Kuisle RJ, Gangat N, et al. Clinical applications and utility of a precision medicine approach for patients with unexplained cytopenias. Mayo Clin Proc. 2019;94:1753-68.

14. Raghu G, Remy-Jardin M, Myers JL, Richeldi L, Ryerson CJ, Lederer DJ, et al. Diagnosis of idiopathic pulmonary fibrosis. An official ATS/ERS/JRS/ALAT Clinical Practice Guideline. Am J Respir Crit Care Med. 2018;198:e44-e68.

15. Blasco MA. The epigenetic regulation of mammalian telomeres. Nat Rev Genet. 2007;8:299-309.

16. Huda N, Tanaka H, Herbert BS, Reed T, Gilley D. Shared environmental factors associated with telomere length maintenance in elderly male twins. Aging Cell. 2007;6:709-13.

\section{ACKNOWLEDGEMENTS}

Authors acknowledge support from the Mayo Clinic Center for Individualized Medicine. Authors would like to acknowledge and thank the referral testing laboratories (Johns Hopkins Medical Laboratories in Baltimore, MD and RepeatDx in Vancouver, Canada).

\section{AUTHOR CONTRIBUTIONS}

AAM wrote the initial and subsequent drafts of the manuscript, and performed data extraction and analysis. AF performed a part of the data extraction and edited the manuscript. FPV, CWH, CP, NG, WJH, MRL, SGP, JPS, JPU, MB, ECP, SK, HS, SPK, DAS, EWK, PSK, ACR, AYJ, CCK, and MEW contributed patients and edited the manuscript. MMP supervised the study and edited all drafts of the manuscript.

\section{CONFLICT OF INTEREST}

The authors declare no competing interests.

\section{ADDITIONAL INFORMATION}

Supplementary information The online version contains supplementary material available at https://doi.org/10.1038/s41408-021-00564-7.

Correspondence and requests for materials should be addressed to Mrinal M. Patnaik.

Reprints and permission information is available at http://www.nature.com/ reprints

Publisher's note Springer Nature remains neutral with regard to jurisdictional claims in published maps and institutional affiliations.

Open Access This article is licensed under a Creative Commons Attribution 4.0 International License, which permits use, sharing, adaptation, distribution and reproduction in any medium or format, as long as you give appropriate credit to the original author(s) and the source, provide a link to the Creative Commons license, and indicate if changes were made. The images or other third party material in this article are included in the article's Creative Commons license, unless indicated otherwise in a credit line to the material. If material is not included in the article's Creative Commons license and your intended use is not permitted by statutory regulation or exceeds the permitted use, you will need to obtain permission directly from the copyright holder. To view a copy of this license, visit http://creativecommons. org/licenses/by/4.0\%.

(c) The Author(s) 2021 